\title{
Effects of kallikrein-related peptidase 14 gene inhibition by small interfering RNA in ovarian carcinoma cells
}

\author{
RUITAO ZHANG, HUIRONG SHI, ZHIMIN CHEN, WEI FENG, HAILING ZHANG and KAIYUAN WU
}

Department of Obstetrics and Gynaecology, First Affiliated Hospital, Zhengzhou University, Zhengzhou 450052, P.R. China

Received July 24, 2011; Accepted September 30, 2011

DOI: $10.3892 / \mathrm{mmr} .2011 .625$

\begin{abstract}
Kallikrein-related peptidase 14 (KLK14) is a member of the tissue kallikrein family of proteases, which are associated with the pathogenesis of malignant tumors and are overexpressed in ovarian carcinoma. However, the mechanism through which KLK14 is implicated in ovarian cancer remains unclear. The aim of the present study was to investigate the effects of KLK14 gene inhibition by small interfering RNA (siRNA) on the growth, apoptosis and invasion of ovarian carcinoma cells in vitro. KLK14 siRNA was transiently transfected into SK-OV-3 and OVCAR-3 ovarian carcinoma cells for $48 \mathrm{~h}$. The expression of KLK14 was determined by reverse transcriptionpolymerase chain reaction (RT-PCR) and Western blot analysis. Cell proliferation, apoptosis and invasion were examined by MTT, flow cytometry and Matrigel assay, respectively. The expression of survivin, caspase 9, cleaved caspase 3 and MMP2 protein was measured by Western blot analysis. The expression of KLK14 was significantly downregulated by siRNA in SK-OV-3 and OVCAR-3 cells at both the mRNA and protein levels. Following transfection with KLK14 siRNA, cell growth and invasion were significantly suppressed, and cell apoptosis was markedly induced. The expression of survivin and MMP2 was decreased, while the espression of caspase 9 and cleaved caspase 3 was increased. These results indicate that KLK14 is implicated in the malignant behavior of ovarian carcinoma cells in vitro, and that KLK14 may serve as a target for therapy of ovarian carcinoma.
\end{abstract}

\section{Introduction}

Ovarian cancer is a malignant tumor which is associated with the highest mortality among female genital system cancers and is considered to be a hormone-associated malignancy. The 5-year survival rate of early-stage ovarian cancer patients is approximately $90 \%$, but less than $30 \%$ in late-stage patients.

Correspondence to: Professor Huirong Shi, Department of Obstetrics and Gynaecology, First Affiliated Hospital, Zhengzhou University, No. 1 Jian She Road, Zhengzhou, Henan 450052, P.R. China

E-mail: huirongshi_zzu@yahoo.com.cn

Key words: kallikrein-related peptidase 14, small interfering RNA, ovarian carcinoma, therapy target
Moreover, more than $70 \%$ of ovarian cancer patients are found to present with late-stage disease at the initial diagnosis (1).

KLK14, also known as kallikrein-like protein 6, was found to be overexpressed in various malignant epithelial tissues and biological fluids, particularly in steroid hormone-related malignancies, including breast cancer, ovarian carcinoma and prostate cancer (2-4). Abnormal expression of KLK14 was found to be related with poor prognosis and has been demonstrated to be a potential biomarker for these malignancies $(4,5)$. However, the mechanism through which KLK14 is implicated in ovarian carcinoma remains unclear.

In the present study, small interfering RNA (siRNA) was used to inhibit endogenous KLK14 gene expression in SK-OV-3 and OVCAR-3 cells. The effects of KLK14 inhibition on the biological behavior of SK-OV-3 and OVCAR-3 cells were observed, and the expression of survivin, caspase 9, cleaved caspase 3 and MMP2 protein was measured.

\section{Materials and methods}

Cell culture and siRNA transfection. Human ovarian carcinoma SK-OV-3 and OVCAR-3 cells (with high levels of KLK14 as detected in our preliminary research) were obtained from the Chinese Academy of Sciences Cell Bank (Shanghai, China), cultured in DMEM/F12 and DMEM media supplemented with $10 \%$ fetal bovine serum (FBS) at $37^{\circ} \mathrm{C}$ in a humidified atmosphere with $5 \% \mathrm{CO}_{2}$. KLK14 siRNA and Transfection Reagent (Santa Cruz Biotechnology, Santa Cruz, CA, USA) were used for siRNA transient transfection, following the manufacturer's instructions. As a negative control, $10 \mu \mathrm{M}$ control siRNA (Santa Cruz Biotechnology) was used. There were three investigation groups in this study: the blank control group (normal cancer cells), negative control group (cancer cells transfected with control siRNA) and KLK14 siRNA group (cancer cells transfected with KLK14 siRNA). Following siRNA transfection for $48 \mathrm{~h}$, cells of each group were harvested for analysis.

Reverse transcription-polymerase chain reaction (RT-PCR). Total RNA was extracted using TRIzol reagent (Invitrogen, Carlsbad, CA, USA) following the manufacturer's instructions. First-strand cDNA was synthesized from $1 \mu \mathrm{g}$ total RNA according to the protocol of the RevertAid First Strand cDNA synthesis kit (Fermentas, EU) using an oligo(dT) primer. Primers used for RT-PCR were as follows: KLK14 sense 5'-ACGCACCCCAACTACAACTC-3', 

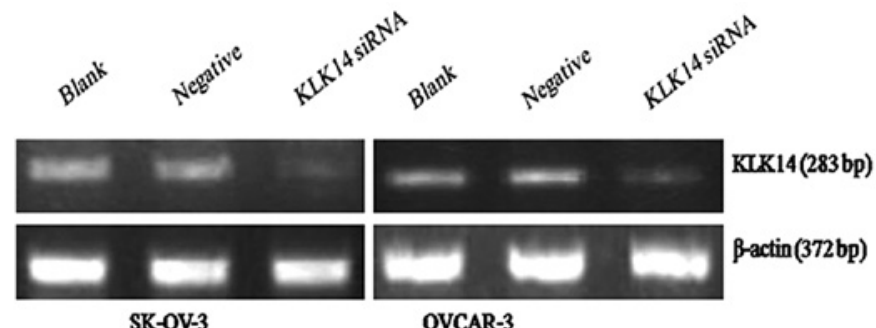

OVCAR-3

C

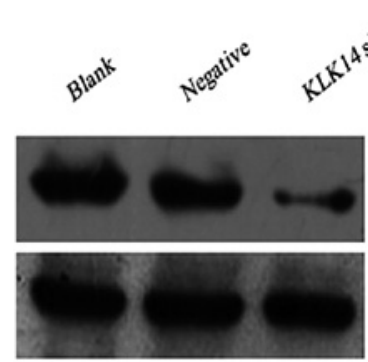

SK-OV-3
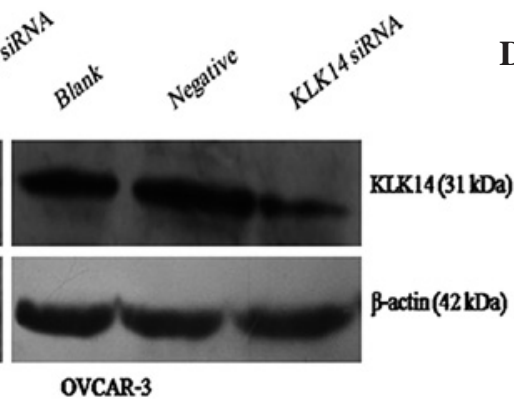

B

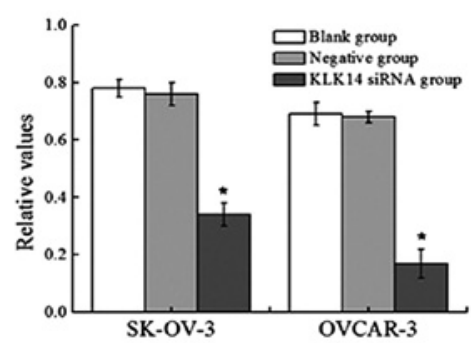

D

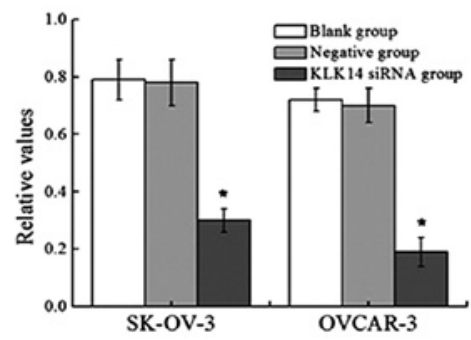

Figure 1. Inhibition of KLK14 expression, as mediated by siRNA in ovarian carcinoma cells. (A) RT-PCR analysis of KLK14 expression in ovarian carcinoma cells. (B) Histograms showing the relative expression levels of KLK14 mRNA. Each bar represents the mean \pm SD. ${ }^{*} \mathrm{P}<0.05$. (C) Western blot analysis of KLK14 protein expression in ovarian carcinoma cells. (D) Histograms showing the relative expression levels of KLK14 protein. Each bar represents the mean \pm SD. ${ }^{*} \mathrm{P}<0.05$.

antisense 5'-GAACTCCTGC-ACAGACCATG-3'; $\beta$-actin sense 5'-ACGCACCCCAACTACAACTC-3', antisense 5'-TCTCCTTAATGTCACGCACGA-3'. PCR cycling parameters (19 cycles) were as follows: denaturation $\left(94^{\circ} \mathrm{C}, 30 \mathrm{sec}\right)$, annealing $\left(58.5^{\circ} \mathrm{C}, 30 \mathrm{sec}\right)$ and extension $\left(72^{\circ} \mathrm{C}, 30 \mathrm{sec}\right)$. Equal amounts of PCR products were electrophoresed on $1.2 \%$ agarose gels and visualized by ethidium bromide staining. $\beta$-actin was used as a control for normalization. The specific bands were analyzed by the Image-Pro Plus 6.0 system.

Western blot analysis. The primary antibodies used in the Western blot analysis, following the manufacturer's instructions, were anti-KLK14 and anti-MMP2 (Santa Cruz Biotechnology), anti- $\beta$-actin, anti-survivin, anti-caspase 9 and anti-cleaved caspase 3 (Beyotime Biotechnology, Haimen, Jiangsu, China). Total cell protein was isolated using cell lysis buffer for Western and IP (Beyotime Biotechnology). Protein concentrations were determined using the bicinchoninic acid (BCA) assay. Equal amounts of protein $(30 \mu \mathrm{g})$ were separated by $10 \%$ sodium dodecyl sulfate polyacrylamide gel electrophoresis (SDS-PAGE) and transferred onto polyvinylidene fluoride (PVDF) membranes. The detection of hybridized protein was performed by using an enhanced chemiluminescence kit; $\beta$-actin was used as a control for normalization. The specific bands were analyzed by the Image-Pro Plus 6.0 system.

MTT assay. Cells were seeded $1 \times 10^{4}$ per well into 96 -well plates and cultured continuously for 5 days. MTT reagent $(10 \mu \mathrm{l})$ (5 g/l; Sigma, St. Louis, MO, USA) was added every day at the same time. Cells were incubated for $4 \mathrm{~h}$ and then the former medium was aspirated and $150 \mu 1$ DMSO was added. The absorbance of samples was measured by a microplate spectrophotometer (Thermo Spectronic, Madison, WI, USA) at $492 \mathrm{~nm}$. All experiments were carried out in triplicate.
Assessment of apoptosis by flow cytometry. Approximately $1 \times 10^{6}$ cells were prepared into a single cell suspension using phosphate-buffered saline (PBS) solution following the manufacturer's instructions included in the Annexin V-FITC Apoptosis Detection kit (Beyotime Biotechnology). The rate of apoptosis was then analyzed with FACScan (BD Biosciences, San Jose, CA, USA). All experiments were performed in triplicate.

Matrigel invasion assay. Transwell chambers ( $8 \mu \mathrm{m}$ pore size; Millipore, Bedford, MA, USA) covered with Matrigel (BD, Franklin Lakes, NJ, USA) were used to measure cell invasive ability. Approximately $1 \times 10^{5}$ cells were seeded into each upper chamber with $200 \mu \mathrm{l}$ fresh medium without FBS, and $500 \mu \mathrm{l}$ medium with $20 \%$ FBS was added into each lower chamber. After $12 \mathrm{~h}$, the cells were fixated with methanol for $5 \mathrm{~min}$ and stained with hematoxylin for $30 \mathrm{~min}$. The upper chamber was cleaned and inverted, and cell numbers on the lower membrane were counted under a high power lens $(x 400)$ in five random visual fields.

Statistical analysis. All of the values are expressed as the mean \pm standard deviation (SD). Statistical analysis was performed by one-way analysis of variance (ANOVA) and Bonferroni test using SPSS 17.0. Differences were considered significant at a P-value $<0.05$.

\section{Results}

Downregulation of KLK14 expression by siRNA. RT-PCR and Western blot analysis were used to measure the expression of KLK14 in each group. Compared to the control groups, the expression of KLK14 mRNA and protein was markedly decreased in the KLK14 siRNA group, both in the SK-OV-3 and OVCAR-3 cells (Fig. 1). 
A

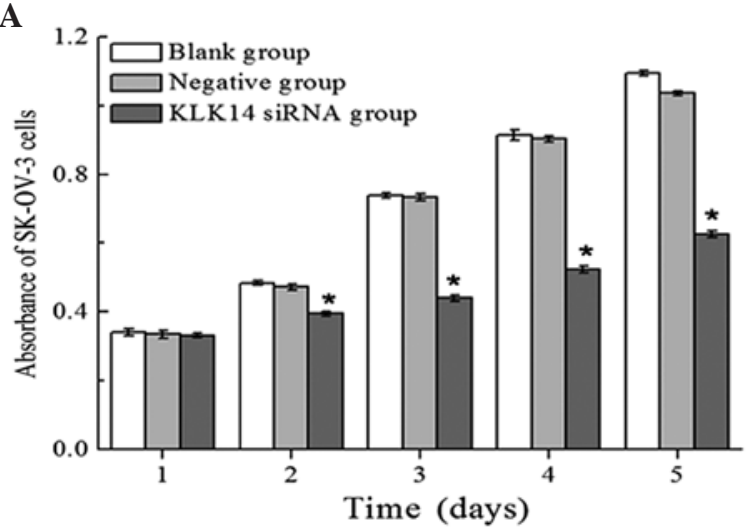

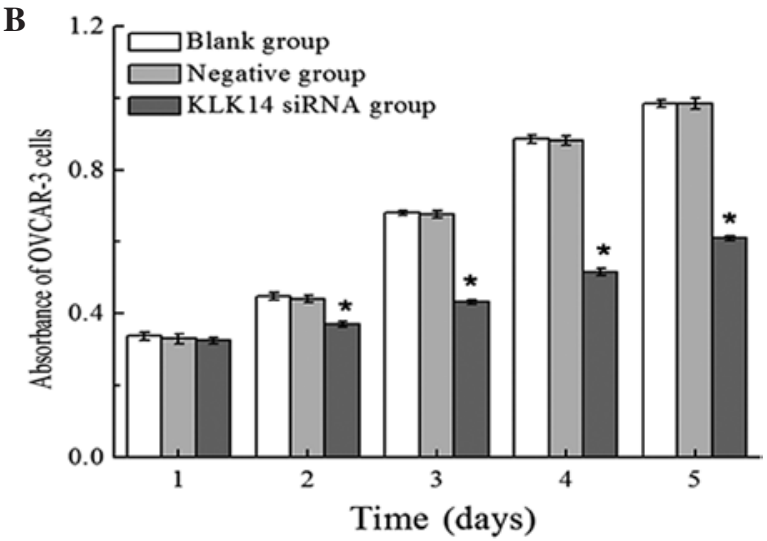

Figure 2. Inhibition of KLK14 expression, as mediated by siRNA, suppresses the growth of ovarian carcinoma cells. (A) Results of the MTT assay showing inhibition of SK-OV-3 cell growth by KLK14 siRNA. Each bar represents the mean \pm SD. (B) Results of the MTT assay showing inhibition of OVCAR-3 cell growth by KLK14 siRNA. Each bar represents the mean \pm SD. ${ }^{*} \mathrm{P}<0.05$.

Blank
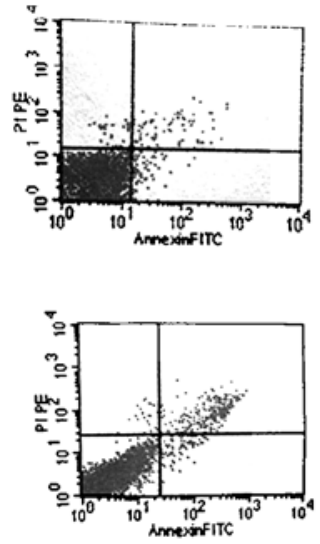

Negative
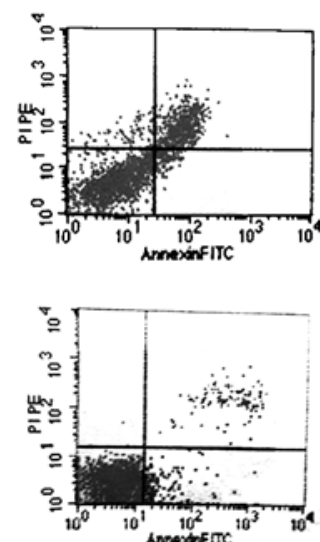

KLK14 siRNA

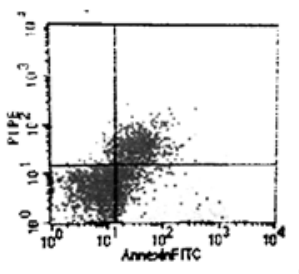

SK-OV-3

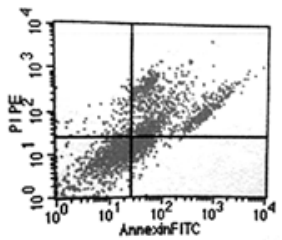

OVCAR-3

Figure 3. Inhibition of KLK14 expression, as mediated by siRNA, induces the apoptosis of ovarian carcinoma cells as detected using flow cytometry.

Suppression of cell growth by KLK14 siRNA. The absorbance of each group as detected by MTT assay is shown in Fig. 2. Significant effects of cell growth inhibition were observed in the KLK14 siRNA group, both in the SK-OV-3 and OVCAR-3 cells from the second day, whereas no differences were detected between control groups.

Cell apoptosis induced by KLK14 siRNA. The apoptosis rates were $3.32 \pm 0.12,7.26 \pm 0.09$ and $17.02 \pm 0.15 \%$ for the SK-OV-3 cells, and $3.69 \pm 0.07,7.82 \pm 0.09$ and $14.67 \pm 0.07 \%$ for the OVCAR-3 cells in the blank control group, negative control group and the KLK14 siRNA group, respectively (Fig. 3). Following KLK14 siRNA transfection, the rates of apoptosis were markedly increased, both in the SK-OV-3 and OVCAR-3 cells.

Cell invasion inhibited by KLK14 siRNA. The number of cells which infiltrated through the Matrigel was indicative of the invasive potential of the ovarian carcinoma cells. The number of cells on the lower membrane of the Transwell chamber in the KLK14 siRNA group was decreased dramatically by KLK14 siRNA, both in the SK-OV-3 and OVCAR-3 cells (Fig. 4).

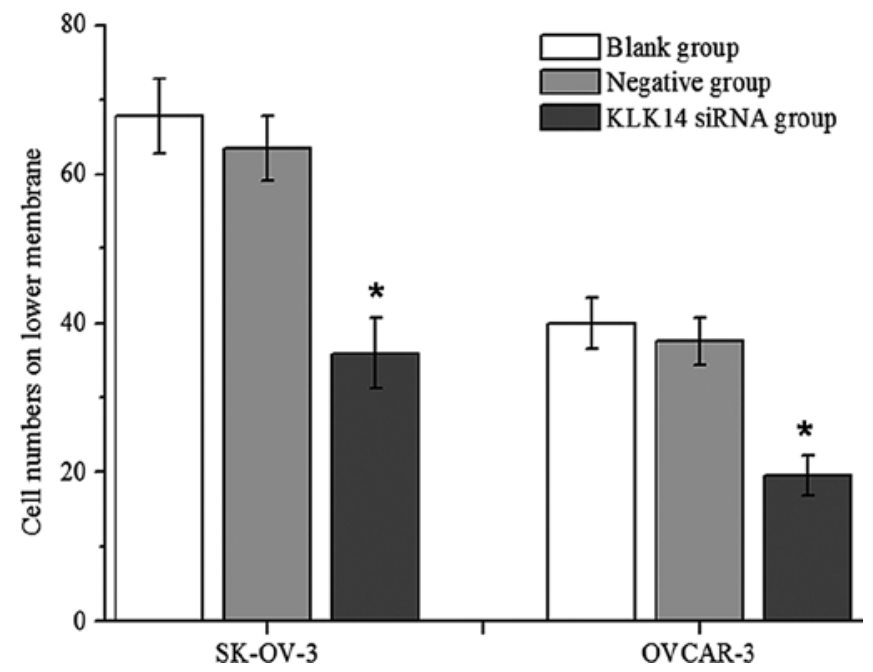

Figure 4. Inhibition of KLK14 expression, as mediated by siRNA, suppresses the invasive ability of ovarian carcinoma cells. Histograms showing the number of cells on the lower membrane which infiltrated the Matrigel. Each bar represents the mean \pm SD. ${ }^{*} \mathrm{P}<0.05$. 


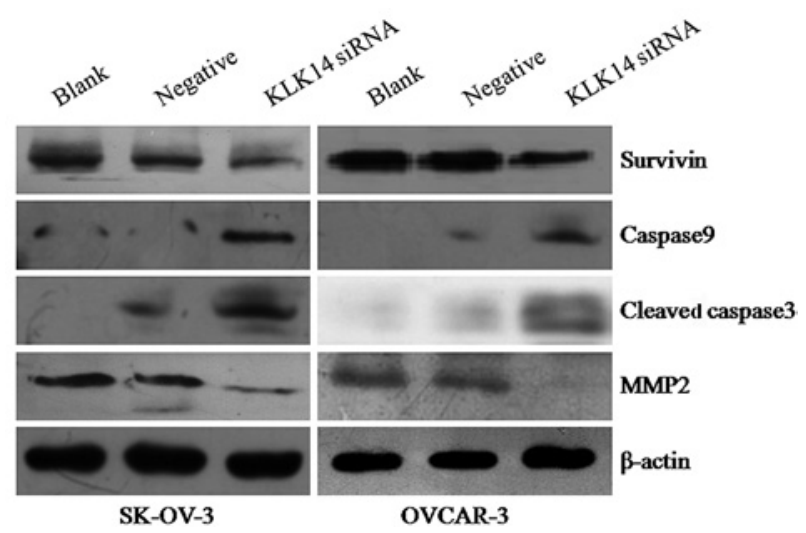

Figure 5. Expression of survivin, caspase 9, cleaved caspase 3 and MMP2 protein as detected by Western blot analysis in the ovarian carcinoma cells. Following inhibition of KLK14 expression, as mediated by siRNA, various alterations in the expression of these proteins were observed.

Expression of survivin, caspase 9, cleaved caspase 3 and MMP2 protein. The results of the Western blot analysis revealed that the expression of survivin and MMP2 was decreased, while the expression of caspase 9 and cleaved caspase 3 was increased by KLK14 siRNA transfection, both in the SK-OV-3 and OVCAR-3 cells (Fig. 5).

\section{Discussion}

The KLK14 gene maps to chromosome 19q13.3-q13.4 and contains seven exons (two untranslated and five coding exons) and six intervening introns (6). The KLK family is implicated in the regulation of numerous cancer-related processes including invasion, metastasis, angiogenesis and tumor growth (7). The targeting of the extracellular matrix (ECM) proteins by the KLKs is considered to be associated with the increased aggressiveness of tumor cells (8).

KLK14 has trypsin- and chymotrypsin-like activity, and is capable of hydrolyzing laminin $\alpha-5$ and collagen IV, which are the major components of the ECM, and may be closely associated with the aggressive nature of malignancies (9). KLK14 was reported to be associated with a number of malignancies, particularly endocrine-related cancers, including breast and testicular cancer $(10,11)$. Recently, KLK14 was also discovered to be upregulated in lung cancer, colorectal cancer and salivary gland tumors (12-14).

In the present study, RT-PCR and Western blot analysis results revealed that the expression of KLK14 was downregulated effectively by KLK14 siRNA in SK-OV-3 and OVCAR-3 cells. Cell growth and invasion were markedly suppressed, and the cell apoptosis rate was increased following KLK14 inhibition. Therefore, this study demonstrated that inhibition of KLK14 by siRNA is capable of suppressing the malignant behavior of ovarian carcinoma cells in vitro.

Survivin, caspase 9, caspase 3 and MMP2 are the most important response proteins associated with cell division, apoptosis and tumor aggressiveness which receive upstream signals to induce cell division, apoptosis and ECM degradation (15-17). According to the results of the Western blot analysis in the present study, expression of survivin and MMP2 was markedly decreased, while expression of caspase 9 and cleaved caspase 3 was markedly increased as a consequence of KLK14 inhibition. These results indicate that the inhibition of cell growth, activation of the apoptosis cascade and suppression of the invasive potential of ovarian carcinoma cells as induced by KLK14 siRNA may be closely related with survivin, caspase 9, caspsae 3 and MMP2.

In conclusion, this study indicates that KLK14 may be implicated in the malignant behavior of ovarian carcinoma cells in vitro and KLK14 may serve as a therapy target for ovarian carcinoma.

\section{References}

1. Parkin DM, Bray F, Ferlay J and Pisani P: Global cancer statistics, 2002. CA Cancer J Clin 55: 74-108, 2005.

2. Yousef GM, Borgoño CA, Scorilas A, et al: Quantitative analysis of human kallikrein gene 14 expression in breast tumours indicates association with poor prognosis. Br J Cancer 87: 1287-1293, 2002

3. Yousef GM, Fracchioli S, Scorilas A, et al: Steroid hormone regulation and prognostic value of the human kallikrein gene 14 in ovarian cancer. Am J Clin Pathol 119: 346-355, 2003.

4. Yousef GM, Stephan C, Scorilas A, et al: Differential expression of the human kallikrein gene 14 (KLK14) in normal and cancerous prostatic tissues. Prostate 56: 287-292, 2003.

5. Borgoño CA, Grass L, Soosaipillai A, et al: Human kallikrein 14: a new potential biomarker for ovarian and breast cancer. Cancer Res 63: 9032-9041, 2003

6. Yousef GM, Magklara A, Chang A, Jung K, Katsaros D and Diamandis EP: Cloning of a new member of the human kallikrein gene family, KLK14, which is down-regulated in different malignancies. Cancer Res 61: 3425-3431, 2001.

7. Borgoño CA and Diamandis EP: The emerging roles of human tissue kallikreins in cancer. Nat Rev Cancer 4: 876-890, 2004.

8. Borgoño CA, Michael IP and Diamandis EP: Human tissue kallikreins: physiologic roles and applications in cancer. Mol Cancer Res 2: 257-280, 2004.

9. Felber LM, Borgoño CA, Cloutier SM, et al: Enzymatic profiling of human kallikrein 14 using phage-display substrate technology. Biol Chem 386: 291-298, 2005.

10. Papachristopoulou G, Avgeris M, Charlaftis A and Scorilas A Quantitative expression analysis and study of the novel human kallikrein-related peptidase 14 gene (KLK14) in malignant and benign breast tissues. Thromb Haemost 105: 131-137, 2011.

11. Luo LY, Yousef G and Diamandis EP: Human tissue kallikreins and testicular cancer. APMIS 111: 225-233, 2003.

12. Planque C, Bléchet C, Ayadi-Kaddour A, et al: Quantitative RT-PCR analysis and immunohistochemical localization of the kallikrein-related peptidases 13 and 14 in lung. Biol Chem 389: 781-786, 2008.

13. Talieri M, Li L, Zheng Y, et al: The use of kallikrein-related peptidases as adjuvant prognostic markers in colorectal cancer. Br J Cancer 100: 1659-1665, 2009.

14. Hashem NN, Mara TW, Mohamed M, et al: Human kallikrein 14 (KLK14) expression in salivary gland tumors. Int J Biol Markers 25: 32-37, 2010.

15. Altieri DC: Survivin, versatile modulation of cell division and apoptosis in cancer. Oncogene 22: 8581-8589, 2003.

16. Alenzi FQ, Lotfy M and Wyse R: Swords of cell death: caspase activation and regulation. Asian Pac J Cancer Prev 11: 271-280, 2010.

17. Orlichenko LS and Radisky DC: Matrix metalloproteinases stimulate epithelial-mesenchymal transition during tumor development. Clin Exp Metastasis 25: 593-600, 2008. 